This document is confidential and is proprietary to the American Chemical Society and its authors. Do not copy or disclose without written permission. If you have received this item in error, notify the sender and delete all copies.

\title{
Freezing Titanium Carbide (MXenes) Aqueous Dispersions for Ultra-long-term Storage
}

\begin{tabular}{|r|l|}
\hline Journal: & ACS Applied Materials \& Interfaces \\
\hline Manuscript ID & am-2020-067285.R1 \\
\hline Manuscript Type: & Article \\
\hline Aute Submitted by the & 06-Jun-2020 \\
\hline Complete List of Authors: & $\begin{array}{l}\text { Zhang, Jizhen; Deakin University, IFM } \\
\text { Kong, Na; Deakin University } \\
\text { Hegh, Dylan; Deakin University } \\
\text { Usman, Ken; Deakin University } \\
\text { Guan, Guangwu; Deakin University } \\
\text { Qin, Si (Alex); Deakin University } \\
\text { Jurewicz, Izabela; University of Surrey, Physics } \\
\text { Yang, Wenrong; Deakin University Faculty of Science Engineering and } \\
\text { Built Environment, School of Life and Environmental Sciences } \\
\text { Razal, Joselito; Deakin University, Institute for Frontier Materials }\end{array}$ \\
\hline
\end{tabular}

\section{SCHOLARONE" Manuscripts}




\title{
Freezing Titanium Carbide (MXenes) Aqueous
}

\section{Dispersions for Ultra-long-term Storage}

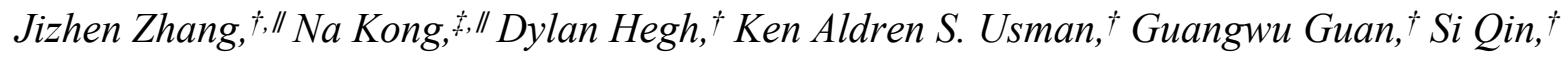 \\ Izabela Jurewicz, ${ }^{\S}$ Wenrong Yang,,+ Joselito M. Razal ${ }^{\dagger}, *$ \\ †Institute for Frontier Materials, Deakin University, Geelong, VIC 3216, Australia. \\ \$School of Life and Environmental Sciences, Deakin University, Geelong, VIC 3216, Australia \\ §Faculty of Engineering and Physical Sciences, University of Surrey, Guildford, Surrey GU2 \\ 7XH, United Kingdom.
}

KEYWORDS: MXene, oxidation, low-temperature, freezing, long-term storage

\begin{abstract}
Two-dimensional titanium carbide $\left(\mathrm{Ti}_{3} \mathrm{C}_{2} \mathrm{~T}_{\mathrm{x}}\right)$, or MXene, is a new nanomaterial that has attracted increasing interest due to its metallic conductivity, good solution processability, and excellent energy storage performance. However, $\mathrm{Ti}_{3} \mathrm{C}_{2} \mathrm{~T}_{\mathrm{x}} \mathrm{MXene}$ flakes suffer from degradation through oxidation due to prolonged exposure to oxygenated water. Preventing the occurrence of oxidation i.e. the formation of $\mathrm{TiO}_{2}$ particles, was found to be the crucial to maintain MXene quality. In present work, we found that freezing aqueous MXene dispersions at low-temperature can
\end{abstract}


effectively prevents the formation of $\mathrm{TiO}_{2}$ nanoparticles at the flake edge, which is known as the early stage of oxidation. The $\mathrm{Ti}_{3} \mathrm{C}_{2} \mathrm{~T}_{\mathrm{x}}$ flakes in frozen dispersion remains consistent in morphology and elemental composition for over 650 days, compared with freshly synthesized MXene; which in contrast, exhibits flake edge degradation within two days when stored at room temperature. This result suggests that freezing MXene dispersion dramatically postpones the oxidation of MXene flakes and the stored MXene dispersion can be treated as freshly prepared MXene. This work not only fundamentally fulfilled the study on temperature dependence of MXene oxidation, but has also demonstrated a simple method to extend the shelf life of MXene aqueous dispersion to years, which will be a cornerstone for large scale production of MXene and ultimately benefit the research on MXenes.

\section{INTRODUCTION}

MXenes are a new class of 2D nanomaterials that have emerged from a large family of transition metal carbides, nitrides and carbonitrides denoted by the general formula $M_{n+1} X_{n} T_{x}(n=1-4)$, where $\mathrm{M}$ is an early transition metal group, $\mathrm{X}$ is carbon and/or nitrogen, and $\mathrm{T}_{\mathrm{x}}$ is surface terminal groups $(-\mathrm{OH},-\mathrm{O}-$, and/or $-\mathrm{F}) \cdot{ }^{1-2}$ MXenes combine $2 \mathrm{D}$ conductive carbide layers with a hydrophilic, primarily hydroxyl-terminated surface. ${ }^{3-7}$ To date, close to 30 MXenes have been successfully synthesized including $\mathrm{Ti}_{3} \mathrm{C}_{2} \mathrm{~T}_{\mathrm{x}}, \mathrm{Ti}_{2} \mathrm{CT}_{\mathrm{x}}$, and $\mathrm{Nb}_{4} \mathrm{C}_{3} \mathrm{~T}_{\mathrm{x}}{ }^{8-9}$ Of the various MXenes, $\mathrm{Ti}_{3} \mathrm{C}_{2} \mathrm{~T}_{x}$ is of particular interest, exhibiting the highest capacitance $\left(\sim 1500 \mathrm{~F} \mathrm{~cm} \mathrm{~cm}^{-3}\right)$ in supercapacitors ${ }^{10}$ and possessing excellent high metallic conductivity $\left(\sim 15000 \mathrm{~S} \mathrm{~cm}^{-1}\right) .{ }^{11}$

Despite the promise of MXenes, one notorious limitation to their widespread implementation is the poor oxidation resistance of MXene flakes in aerated aqueous media, leading to rapid degradation into titanium dioxide $\left(\mathrm{TiO}_{2}\right) \cdot{ }^{5-6,12-13}$ By summarizing previous studies, as shown in 
Figure 1a, the MXene oxidation process can be divided into three stages: firstly (I) the formation of $\mathrm{TiO}_{2}$ at the MXene flake edge; ${ }^{12,14-15}$ followed by (II) expansion of the $\mathrm{TiO}_{2}$ particles from the edge of MXene flake to surface whilst the carbon layer is retained; ${ }^{16-17}$ and finally (III) the carbon layers are etched by hydroxyl radicals generated by the surrounding $\mathrm{TiO}_{2}$ and the $\mathrm{MXene}$ flakes is fully converted to amorphous $\mathrm{TiO}_{2} \cdot{ }^{12,18}$ As a consequence of the oxidation of MXenes, both the electrical conductivity and passivation of the reactive interface are impaired, decreasing the energy storage performance. Thus, maintaining the intact of MXene flakes is a critical demand for the application that requires aqueous solutions of MXene, such as filtration films, ${ }^{19}$ wet-spinning fibers, ${ }^{20}$ hydrogel/aerogel preparation, ${ }^{21-22}$ and aqueous-based supercapacitors. ${ }^{23}$

Investigations into the preservation of MXene flakes have demonstrated that the oxidation rate was influenced by the surrounding media, ${ }^{13,24}$ and it can be slowed down by using organic solvents, ${ }^{18,24}$ air-free water, ${ }^{12}$ antioxidant,${ }^{25}$ or polymer media. ${ }^{13}$ For example, both Seyedin et al. ${ }^{18}$ and Maleski et al. ${ }^{24}$ have reported that using organic solvents, such as dimethyl sulfoxide, ethanol, and N-methyl-pyrrolidone, to replacing water can extend the shelf time of $\mathrm{Ti}_{3} \mathrm{C}_{2} \mathrm{~T}_{\mathrm{x}}$ to over 30 days. Green et al. ${ }^{25}$ found that adding sodium L-ascorbate enables $\mathrm{Ti}_{3} \mathrm{C}_{2} \mathrm{~T}_{\mathrm{x}}$ flakes to retain their colloidal stability in water for 21 days. These approaches, however, only reported on short-term ( 30 days) storage of MXenes; additionally, retention of organic solvents and additives in MXene films or fibers leads to a gradual decrease in electrical conductivity with time. ${ }^{26-28}$ Moreover, repeated centrifugation to transfer across MXene sheets into organic solvents, is laborious and a batch process that takes several hours to complete. This process inevitably increases the exposure time of MXene to water and air allowing edge oxidation to occur. Traditionally, the most efficient approach for long-term storage of MXene has been regarded as a powder or film under vacuum as since it is isolated from water and air. However, film and powder requires the assistance of 
intercalating agents and sonication for re-dispersion in water, which both decreases the flake size and significantly lowers the yield of single-layer MXene. ${ }^{29}$ Therefore, long-term (years) storage of MXenes as an aqueous dispersion without any additives while preventing the oxidation is regarded as ideal; however, this has proven challenging. ${ }^{13,30}$

a

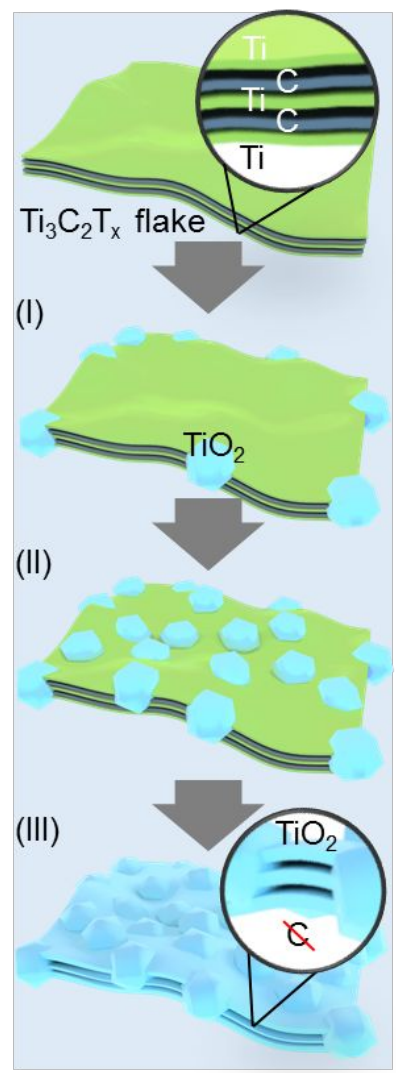

b

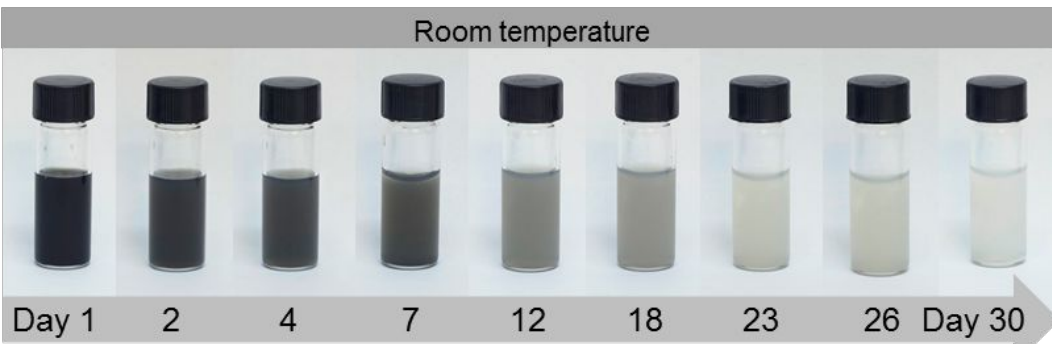

C

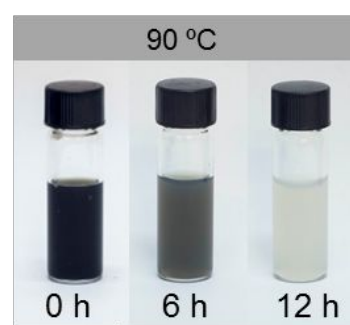

d

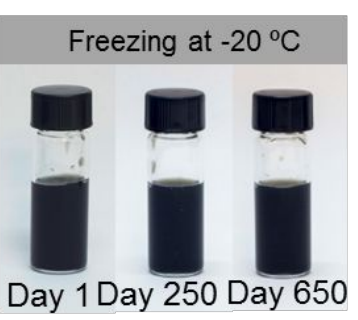

e

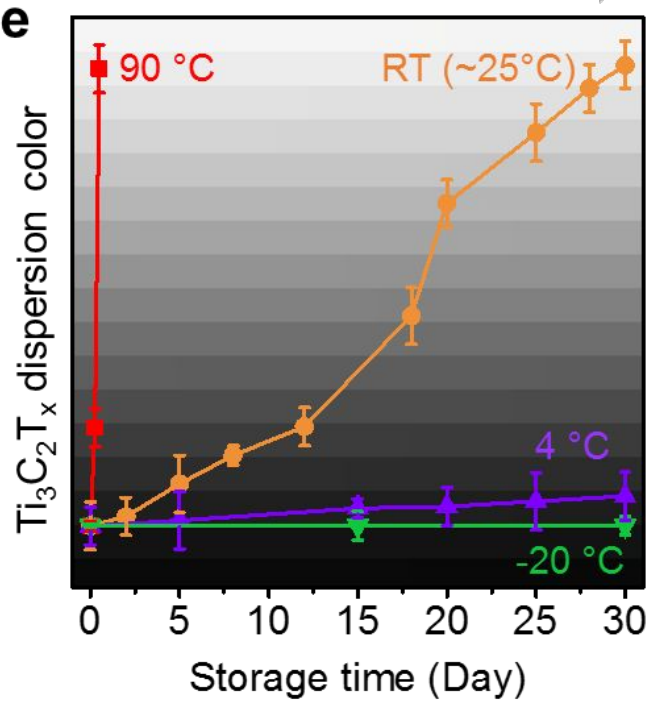

Figure 1. (a) Schematically illustration of the oxidation process of single $\mathrm{Ti}_{3} \mathrm{C}_{2} \mathrm{~T}_{\mathrm{x}}$ MXene flakes in dispersion. Photos of the aqueous MXene dispersion $\left(0.5 \mathrm{mg} \mathrm{mL}^{-1}\right)$ (b) stored at room temperature $\left(\mathrm{RT}, \sim 25^{\circ} \mathrm{C}\right.$ ) over 30 days, (c) incubated in an oven at $90{ }^{\circ} \mathrm{C}$ for 0,6 and 12 hours, and (d) stored at $-20{ }^{\circ} \mathrm{C}$ for 250 and 650 days. (e) Color fading of MXene dispersions stored at different temperatures as a function of storing time, in which the data point was obtained by digital analysis of the photograph of MXene dispersions using Image J. The background is a dark green step-wedge linearly changed from $\mathrm{R}: \mathrm{G}: \mathrm{B}=4: 4: 4$ (bottom) to $\mathrm{R}: \mathrm{G}: \mathrm{B}=$ 247:247:247 (top), which 
works as the y-axis. The error bar is derived from the variation of colors at different positions of the dispersion in the photographs.

Low-temperature preservation is a common strategy to prevent the degradation of biological materials and is widely used in food preservation, biochemical research and medical surgery. ${ }^{31-34}$ Recently, Chae at al. demonstrated that the oxidation of MXene can be significantly delayed by storing at $-80^{\circ} \mathrm{C}$, with the stored MXene showing similar gas response behavior compared with pristine MXene. ${ }^{35}$ However, for wider applicability, a simple and readily available method are still required. Herein, we found that decreasing the storage temperature of aqueous MXene dispersions to $-20{ }^{\circ} \mathrm{C}$ can successfully prolong its shelf-life from tens of days to nearly two years. This temperature can be readily achieved by commonly used freezers in most lab environments. Detailed characterization of the frozen MXene dispersion shows that frozen flakes have intact clean flake edges and high electrical properties compared with the freshly made dispersion. Moreover, it is observed that $\mathrm{TiO}_{2}$ nanoparticles formed at the edge of MXene flakes hinder the close stacking of flakes, which is also responsible for the decrease in conductivity and mechanical properties of MXene films. These findings provide a simple method for storing $\mathrm{Ti}_{3} \mathrm{C}_{2} \mathrm{~T}_{x}$, which may also apply to other MXenes, and it potentially benefits future large-scale synthesis and further MXene studies.

\section{EXPERIMENTAL SECTION}

2.1. Synthesis of $\mathbf{T i}_{3} \mathbf{C}_{\mathbf{2}} \mathbf{T}_{\mathbf{x}}$ dispersions. $\mathrm{Ti}_{3} \mathrm{C}_{2} \mathrm{~T}_{\mathrm{x}}$ was synthesized by a wet-etching process using an etching solution, containing 0.8 g lithium fluoride (LiF, $99 \%$, Sigma-Aldrich Pty Ltd) and 10 $\mathrm{ml}$ of $9 \mathrm{M}$ hydrochloric acid $(\mathrm{HCl})$. Then, $0.5 \mathrm{~g}$ of $\mathrm{Ti}_{3} \mathrm{AlC}_{2}$ powder (MAX phase, $<40 \mu \mathrm{m}$ particle size, Carbon-Ukraine) was slowly added to the etchant and the reaction incubated at room 
temperature for $24 \mathrm{~h}$. The acidic dispersion was washed with deionized (DI) water through several rounds of centrifugation at $3500 \mathrm{rpm}$ until the $\mathrm{pH}$ of the suspension was close to 6 , using a Centrifuge-5810R (Eppendorf AG, Germany), with decantation of the supernatant after each cycle. The single-layer delaminated $\mathrm{Ti}_{3} \mathrm{C}_{2} \mathrm{~T}_{x}$ was collected in the supernatant from multilayer MXene and unreacted MAX phase by further centrifugation at $1500 \mathrm{rpm}$ for $30 \mathrm{~min}$. The as-synthesized dark green supernatant containing single layer $\mathrm{Ti}_{3} \mathrm{C}_{2} \mathrm{~T}_{x}$ flakes was labeled as "Fresh MXene".

2.2. Oxidation study on MXene dispersions. The MXene dispersion $\left(0.5 \mathrm{mg} \mathrm{mL}^{-1}\right)$ was sealed into $4 \mathrm{~mL}$ vials with $3 \mathrm{~mL}$ of dispersion and $1 \mathrm{~mL}$ of air. The vials were then stored at $90{ }^{\circ} \mathrm{C}$, room temperature (RT, $25{ }^{\circ} \mathrm{C}$ ), $4{ }^{\circ} \mathrm{C}$ and $-20^{\circ} \mathrm{C}$. For the samples stored at $-20{ }^{\circ} \mathrm{C}$, the vials that contain $\mathrm{Ti}_{3} \mathrm{C}_{2} \mathrm{~T}_{\mathrm{x}}$ dispersions were wrapped with aluminum foil and placed directly in a conventional freezer without liquid nitrogen treatment. The dispersion stored at RT for two days was labeled as "RT2 " and the frozen sample at $-20^{\circ} \mathrm{C}$ for 650 days was defined as "Frozen- 650 ", in which the number represents the days of storage. The $-20{ }^{\circ} \mathrm{C}$ sample was thawed to room temperature by placing it on a bench for $2 \mathrm{~h}$. After equilibration to room temperature, the dispersion was used for further characterization.

2.3. Characterization. Field emission scanning electron microscope (FESEM) (Zeiss SUPRA 55VP) wasconducted to study the fiber morphology and the cross-section of the LC MXene fibers. Transmission electron microscopy (TEM) characterization was employed on a JEM-2100 to study exfoliated $\mathrm{Ti}_{3} \mathrm{C}_{2} \mathrm{~T}_{\mathrm{X}}$ layer. The X-ray diffraction (XRD) patterns of the fibers were obtained with a powder diffractometer (Rigaku SmartLab, Tokyo, Japan) using $\mathrm{Cu}$ Ka radiation (40 kV, $30 \mathrm{~mA})$ with an X-ray wavelength of $(\lambda) 1.54 \AA$ at a $2 \theta$ scan step of $0.02^{\circ}$ from $3-70^{\circ}$ with a scan time of 14 seconds/step. Dynamic light scattering (DLS) was performed by Zetasizer Nano ZS, Malvern Instruments to measure the size distribution of MXene flakes. DLS measurements were repeated 
for five measurements from each sample. The electrical conductivity of the MXene films was measured using a four-point probe set-up (Ossila Ltd.) with uniform $1 \mathrm{~mm}$ probe spacing. The mechanical properties of MXene films were measured using a universal tensile testing system (Instron $30 \mathrm{kN}$ Tensile Tester) with a $5 \mathrm{~N}$ load cell. Film samples were fixed between the grips with the gap length of $5 \mathrm{~mm}$ and stress and strain data were recorded at an extension rate of 0.5 $\mathrm{mm} \min ^{-1}$.

2.4. Electrochemical test of MXene electrodes. Electrochemistry tests were performed using a three-electrode configuration with carbon rod and $\mathrm{Ag} / \mathrm{AgCl}(3.5 \mathrm{M} \mathrm{KCl})$ electrode as counter and reference electrodes, respectively. $\mathrm{H}_{2} \mathrm{SO}_{4}(1 \mathrm{M})$ solution was used as electrolyte. The working electrode was prepared by drop-casting $3 \mu \mathrm{L}$ of $\mathrm{Ti}_{3} \mathrm{C}_{2} \mathrm{~T}_{\mathrm{x}}$ dispersion on a glassy carbon electrode (diameter of $3 \mathrm{~mm})$. The electrode was then coated with Nafion ${ }^{\circledR} 117$ solution $(\sim 5 \mathrm{wt} . \%, \sim 2 \mu \mathrm{L})$ to prevent the thin MXene film from peeling off. After drying under vacuum at RT for $2 \mathrm{~h}$, MXene electrodes were tested using a Biologic SP-300 electrochemical station at room temperature. EIS measurements were carried out at an open circuit potential by applying an alternating-current voltage with an amplitude of $10 \mathrm{mV}$ in a frequency range of $10 \mathrm{mHz}$ to $1 \mathrm{MHz}$. The long-term cyclic stability test was performed using $\mathrm{CV}$ at $50 \mathrm{mV} \mathrm{s}-1$ for 10,000 cycles. The values of gravimetric capacitance $\left(\mathrm{C}, \mathrm{F} \mathrm{g} \mathrm{g}^{-1}\right)$ of the electrodes were estimated from:

$$
C=\frac{\int I d U}{M v \Delta U}
$$

where $I$ is the current $(\mathrm{A}), v$ is the potential scan rate $\left(\mathrm{V} \mathrm{s}^{-1}\right), \Delta U$ is the potential window $(\mathrm{V})$, and $M$ is the mass of MXene loaded on the electrode, respectively.

\section{RESULTS AND DISCUSSION}


3.1. Synthesis of $\mathbf{T i}_{3} \mathbf{C}_{2} \mathbf{T}_{\mathbf{x}}$ MXene dispersion. $\mathrm{Ti}_{3} \mathrm{C}_{2} \mathrm{~T}_{\mathrm{x}}$ MXene flakes (from here on referred to as "MXene") were synthesized from the $\mathrm{Ti}_{3} \mathrm{AlC}_{2}$ precursor $(0.5 \mathrm{~g})$ by selective etching of the aluminum layer using a mixture of $\operatorname{LiF}(0.8 \mathrm{~g})$ and $\mathrm{HCl}(9 \mathrm{M}, 10 \mathrm{~mL})$ at room temperature $(\mathrm{RT}$, $\sim 25^{\circ} \mathrm{C}$ ) for $24 \mathrm{~h}$. The subsequent intercalation of water and Li ions within the negatively charged surface resulted in a volume increase during washing with ultra-pure water indicates the selfdelamination of multi-layered MXene to few/single layers. Delamination and the removal of the aluminum was confirmed by the down-shifting of the (002) peak and a disappearance of aluminum peak at $2 \theta$ of $39^{\circ}$ on X-ray diffraction (XRD) spectra (Figure S1a). The dispersed, or assynthesized, MXene suspension was dark green in color and the flakes exhibited an average hydrodynamic diameter of $\sim 500 \mathrm{~nm}$ (Figure S1b). These dispersions, referred to as "Fresh MXene" were subsequently stored under various conditions to study their rate of degradation.

Compared with previous studies on MXene oxidation stability, ${ }^{12,14,}{ }^{18}$ we synthesized MXene using a higher molar ratio of $\mathrm{LiF}$ and $\mathrm{Ti}_{3} \mathrm{AlC}_{2}(12: 1$ vs. 7.5:1) and a lower etching temperature (RT vs. $35^{\circ} \mathrm{C}$ ). Etching $\mathrm{Ti}_{3} \mathrm{AlC}_{2}$ in the presence of a high $\mathrm{LiF}$ molar ratio is known to produce readilydelaminated MXene without further sonication. ${ }^{14,36}$ Delamination and/or re-dispersion of MXene with the aid of sonication has been found to cause flake damage and induce oxidation, resulting in lower conductivity. ${ }^{18,}$ 37-38 Likewise, elevated etching temperature also promotes MXene oxidation. ${ }^{16,39}$ It is noteworthy that the frozen MXene dispersion employed in this study allowed us to eliminate the initial oxidation of MXene caused by both sonication and heating.

To assess the difference of this synthetic variation with previously published MXene oxidation studies, ${ }^{12,14,18}$ a dispersion $\left(0.5 \mathrm{mg} \mathrm{mL}^{-1}\right)$ was monitored at room temperature. Oxidation of the MXene dispersion resulted in fading of the solution color from black, or translucent greenish at very low concentrations, to cloudy white upon full MXene oxidation, due to the formation of $\mathrm{TiO}_{2}$ 
crystals at the edges and surface of MXene flakes (Figure 1a). ${ }^{12}$ Although the color of MXene dispersions depend on MXene concentration, color change is typically the proxy used to indicate the oxidation of MXene flakes, as such the same concentration of MXene was used. ${ }^{12,18}$ Fading can be easily observed as early as the second day (RT-2) and continues until the dispersion turns completely white by day 30 (RT-30) (Figure 1b). Oxidation of MXene is indicated by color fading and solution stability was observed to be similar with previous reports on MXene oxidation that used different etching conditions. ${ }^{12,40}$

3.2. Temperature dependence of MXene oxidation rate in aqueous dispersion. To study the temperature dependence of MXene oxidation rate, the as-synthesized MXene dispersions were stored at temperatures from $-20{ }^{\circ} \mathrm{C}, 4{ }^{\circ} \mathrm{C}, 25^{\circ} \mathrm{C}$ and $90{ }^{\circ} \mathrm{C} .-20{ }^{\circ} \mathrm{C}$ was selected as the lowest temperature in our study, which was the readily reached temperature of a general laboratory freezer, aiming to demonstrate the wide applicability of this storage method. The color changes of all samples were plotted on a dark green step-wedge (Figure 1e). MXene dispersions exhibited reduced stability with increasing temperature from 30 days at $20{ }^{\circ} \mathrm{C}$, (Figure 1b) to less than 24 hours at $90{ }^{\circ} \mathrm{C}$ (Figure 1c). In contrast, there was no change in color when the as-synthesized MXene dispersion was frozen at $-20{ }^{\circ} \mathrm{C}$, even after 650 days (Frozen-650, Figure $1 \mathrm{~d}$ ). For the MXene dispersion stored at $4{ }^{\circ} \mathrm{C}$, we observed a significant delay in color change, however oxidation still occurred within 30 days. Those observation suggest that the rate of oxidation increases with temperature. Though irreversible, the rate of oxidation could then be slowed down by lowering the temperature. ${ }^{12}$ From the color changes, freezing the MXene dispersion seems to be the best approach for long term storage of MXene. 

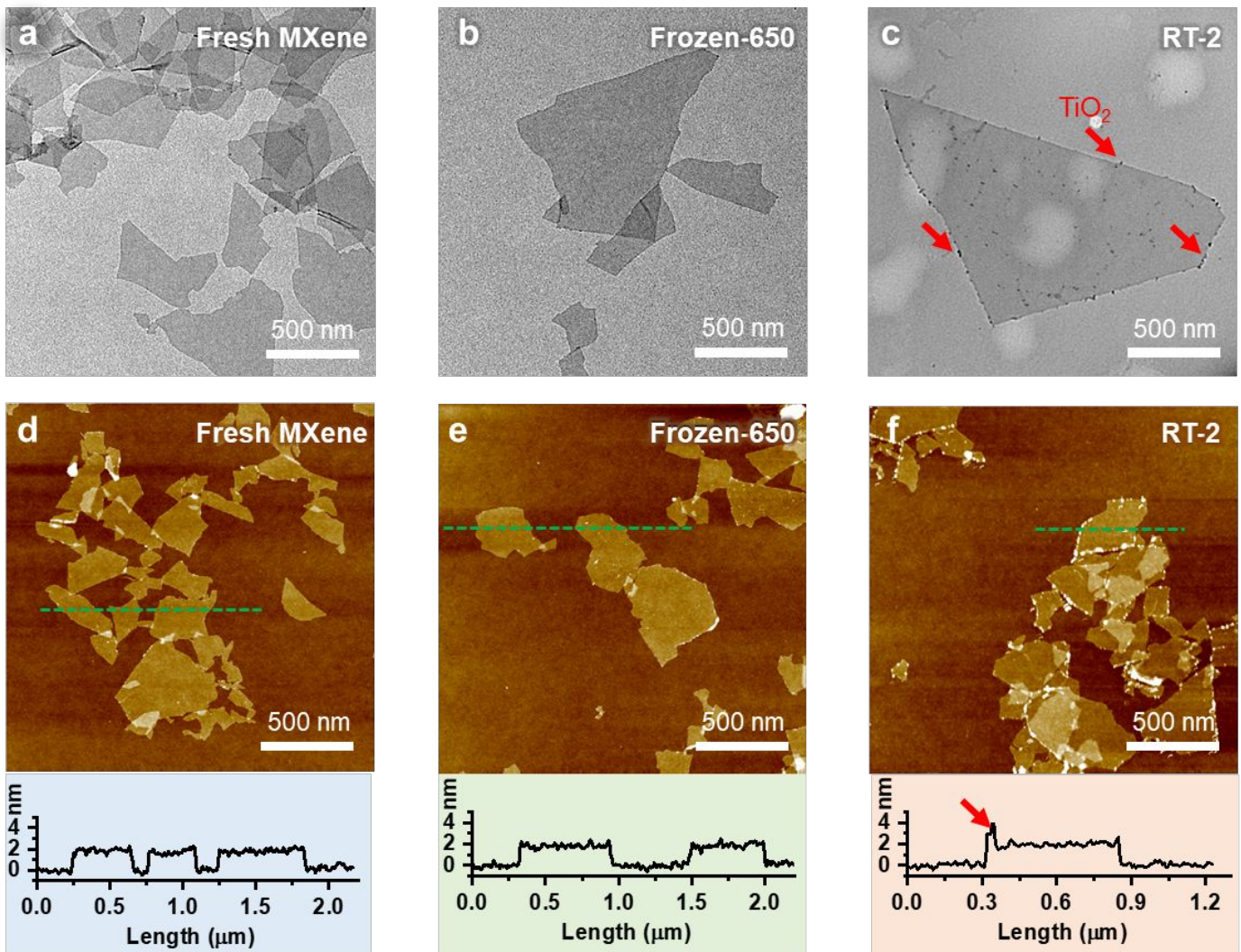

Figure 2. Morphology and structural characterization of $\mathrm{Ti}_{3} \mathrm{C}_{2} \mathrm{~T}_{\mathrm{x}}$ flakes. TEM images of (a) Fresh MXene, (b) Frozen-650, and (c) RT-2 samples. AFM images of (d) Fresh MXene, (e) Frozen-650, and (f) RT-2 samples. Bottom represents the thickness data along the green lines indicated in figures. The red arrow indicates the $\mathrm{TiO}_{2}$ particles at the edge of $\mathrm{MXene}$ flakes.

To investigate the effect of freezing on the oxidation degree of MXene flakes, the morphology changes of MXene flakes taken from Fresh MXene, Frozen-650, and RT-2 were assessed through transmission electron microscopy (TEM) and atomic force microscopy (AFM) studies. Figure 2a and $2 \mathrm{~b}$ show that both Fresh MXene and Frozen-650 exhibited a clean surface at the edge of the MXene flakes. For RT-2, small black dots were observed, indicating the formation of nanoparticles at the edge of MXene flakes, suggesting Stage I oxidation ${ }^{12,14}$ (Figure 2c). The AFM micrographs of $\mathrm{Ti}_{3} \mathrm{C}_{2} \mathrm{~T}_{\mathrm{x}}$ flakes in Fresh MXene show flake thicknesses of $\sim 1.9 \mathrm{~nm}$, indicating the synthesis of 
single layer MXene flakes. ${ }^{14,41} \mathrm{Ti}_{3} \mathrm{C}_{2} \mathrm{~T}_{\mathrm{x}}$ flakes stored after two days at normal laboratory condition (RT-2) exhibited a significant increase in thickness $(\sim 4 \mathrm{~nm})$ at the flake edge while in contrast the Frozen-650 sample showed flakes with clean surfaces (Figure $2 b$ and c), indicating that freezing prevents the formation of $\mathrm{TiO}_{2}$ particles. The DLS data shows a size distribution around 90-100 $\mathrm{nm}$ which were consistent with TEM and AFM images in which small MXene flakes with sizes around $100 \mathrm{~nm}$ could be observed.

To study the changes in the elemental composition of MXene flakes after storage, X-ray photoelectron spectroscopy (XPS) analysis was performed. High resolution XPS spectra of MXene at varying storage conditions were fitted into Ti 2p, C 1s and O 1s core levels (Figure 3). The peak corresponding to the Ti $2 p$ core level was fitted to four doublets of Ti $2 p_{3 / 2}$ and $T i 2 p_{1 / 2}$, namely Ti-C $2 p_{3 / 2}$, Ti-C $2 p_{1 / 2}, \operatorname{Ti}(I I) 2 p_{3 / 2}, \operatorname{Ti}(I I) 2 p_{1 / 2}, \operatorname{Ti}(I I I) 2 p_{3 / 2}, \operatorname{Ti}(I I I) 2 p_{1 / 2} \operatorname{Ti}(I V) 2 p_{3 / 2}$ and Ti(IV) $2 \mathrm{p}_{1 / 2}$ located at $\sim 454.9,460.8,455.9,461.9,457.6,462.9 \mathrm{eV}, 458.5 \mathrm{eV}$ and $464.1 \mathrm{eV}$, respectively (Figure 3a, $\mathrm{d}$ and $\mathrm{g})$. The $\mathrm{C} 1 \mathrm{~s}$ core was fitted to four peaks of C-Ti $(281.8 \mathrm{eV})$, C-Ti-O (282.0 eV), C-C $(284.4 \mathrm{eV})$ and C-O $(286.0 \mathrm{eV})$. The O 1s core was assigned to C-Ti-OH 1s, C-Ti-Ox and O-Ti-O 1s located at $\sim 532.7,529.6$ and $530.6 \mathrm{eV}$, respectively (Figure $3 \mathrm{c}$, f and i). Fitted peaks of the Ti 2p, C 1s and O 1s core levels were all comparable to the previously reported XPS spectra of MXene in existing literature. ${ }^{42-45}$ Fresh MXene and Frozen-650 showed vary similar shape in the Ti $2 p$ region while the Ti-O $2 p_{3 / 2}$ peak exhibited a slight increase in RT-2 spectra, suggesting the formation of $\mathrm{TiO}_{2}$ nanoparticles during Stage I oxidation. The C 1s spectra of all samples showed neglectable change, suggesting the carbon layer in MXene is not decomposed at early stage of oxidation. Furthermore, the $\mathrm{O}$ 1s spectra were relatively similar between Fresh MXene and Frozen-650 samples, whereas the RT-2 showed dramatic increase in the ratio between O-Ti- 
$\mathrm{O}$ 1s and $\mathrm{C}-\mathrm{Ti}-\mathrm{OH}$ 1s peaks (Figure 3), suggesting that the frozen sample retains its elemental composition even after storing for $\sim 2$ years while oxidation occurs within two days for room temperature MXene.
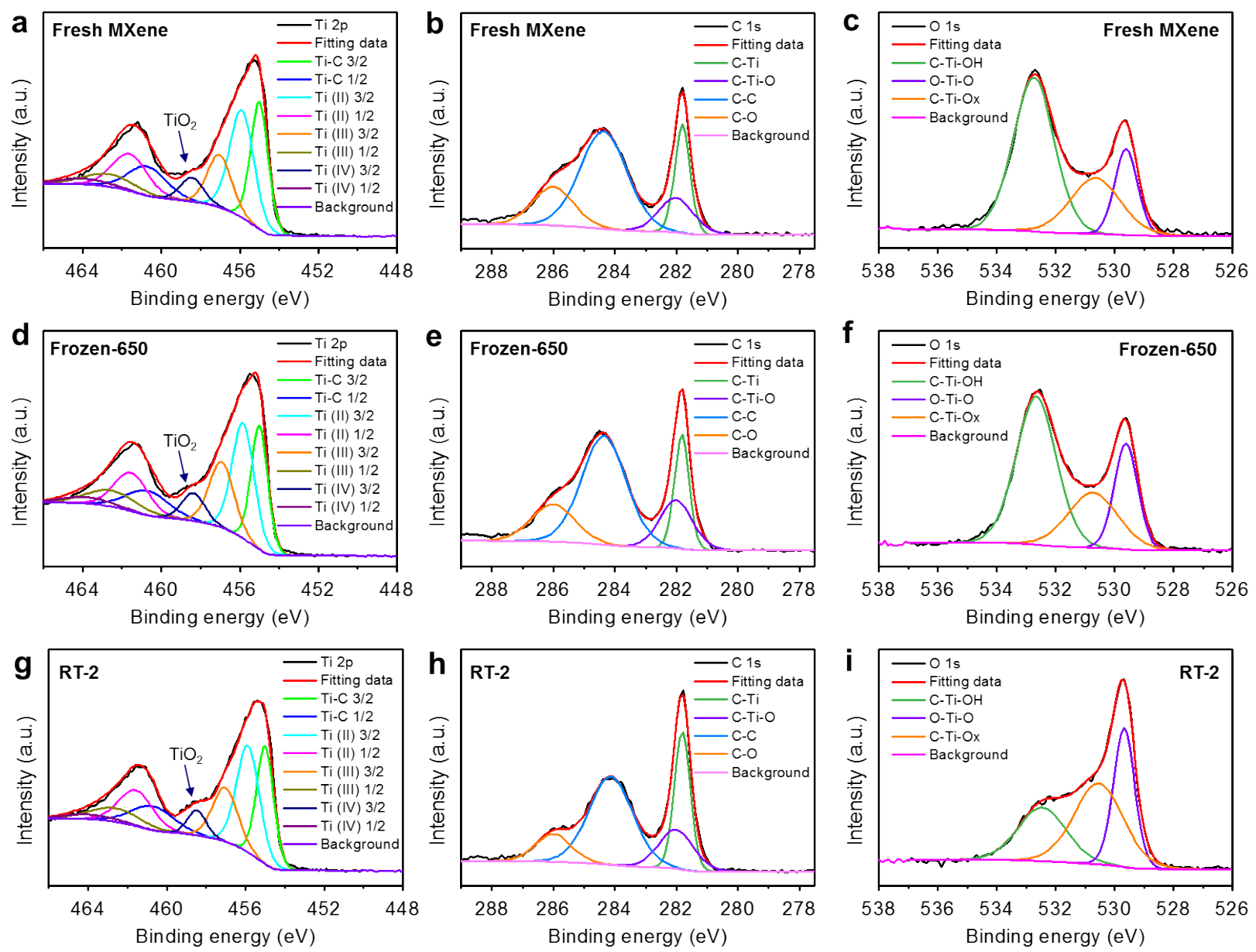

Figure 3. High resolution Ti 2p, C 1s and $\mathrm{O}$ 1s XPS spectra of $\mathrm{Ti}_{3} \mathrm{C}_{2} \mathrm{~T}_{\mathrm{x}}$ films prepared from (a-c) Fresh MXene, (d-f) Frozen-650 and (g-i) RT-2 dispersions.

3.3. Effect of storing conditions on film properties. Free-standing MXene films of similar thicknesses (3.6 to $3.8 \mu \mathrm{m}$ ) were prepared using vacuum-assisted filtration. All films (Fresh MXene, Frozen-650, and RT-2) showed good flexibility (Figure S2), suggesting the early stage 
oxidation, such as that observed in RT-2 dispersions, has negligible effect on the processability using the vacuum assisted filtration method. Quantitative assessment of the mechanical properties of the films revealed that the tensile strength of RT-2 film $(49.4 \pm 3.1 \mathrm{MPa})$ is half of that of Fresh MXene film (84.2 $\pm 3.7 \mathrm{MPa})$ and Frozen-650 film (80.9 $\pm 4.3 \mathrm{MPa})$. The Young's Modulus also decreased from $4.9 \pm 0.6 \mathrm{GPa}$ (Fresh MXene film) and 4.2 $\pm 0.4 \mathrm{GPa}$ (Frozen-650 film) to $2.5 \pm$ 0.2 GPa for RT-2 film (Figure 4a). The electrical conductivity of these films (Figure 4b) showed that the films composed of Frozen-650 and Fresh MXene have higher conductivity of $5608 \pm 302$ $\mathrm{S} \mathrm{cm}^{-1}$ and $5480 \pm 285 \mathrm{~S} \mathrm{~cm}^{-1}$, compared to the RT-2 film $\left(4347 \pm 217 \mathrm{~S} \mathrm{~cm}^{-1}\right)$. These results suggest that the electrical conductivity of MXene films prepared from RT-2 dispersion were significantly compromised, whereas Frozen-650 has almost the same performance compared to that of Fresh MXene.

To understand the stacking structure of MXene flakes in films, XRD spectra and morphology were assessed. Based from the XRD spectra of vacuum filtered films of Fresh MXene, Frozen650, and RT-2 (Figure 4c), all films showed the same peak position for (002) peak $\sim 6.9^{\circ}$, attributing to lamellar layer spacing of $1.2 \mathrm{~nm}$. The full width at half maximum (FWHM) of this (002) peak for Fresh MXene and Frozen- 650 are $0.62^{\circ}$ and $0.65^{\circ}$, respectively, while the FWHM increased to $1.25^{\circ}$ for RT-2 film. According to Scherrer's equation, the increase in FWHM indicates that MXene flakes in the RT-2 film has irregular orientation and the increased intensity at low 2 theta indicates larger d-spacing between MXene flakes. Considering the formation of $\mathrm{TiO}_{2}$ particles at the edges of flakes, it can be an important reason for the decrease of conductivity and mechanical strength of RT-2 film. The SEM micrographs of the cross-section of MXene films at the same magnification revealed that Fresh MXene and Frozen-650 films exhibit close stacking of the MXene along the film plane, while RT-2 film shows loose packing with small particles between 
MXene flakes (Figure 4d-f). These small particles can be attributed to $\mathrm{TiO}_{2}$, as suggested by Energy Dispersive X-ray (EDX) data on MXene films showing slightly increased oxygen content from 16.6 wt.\% for Fresh MXene film to 18.6 wt.\% for RT-2 film (Figure S3). Due to the increased layer spacing, RT-2 film showed a lower density of $3.2 \mathrm{~g} \mathrm{~cm}^{-3}$ compared to that of Fresh MXene $\left(3.4 \mathrm{~g} \mathrm{~cm}^{-3}\right)$. We proposed the following structural models of $\mathrm{Ti}_{3} \mathrm{C}_{2} \mathrm{~T}_{\mathrm{x}}$ films prepared using fresh $\mathrm{Ti}_{3} \mathrm{C}_{2} \mathrm{~T}_{\mathrm{x}}$ and oxidized RT-2 dispersions. Fresh MXene flakes possess a uniformly smooth flat surface, and forms closely stacked layers when filtered into a film. In contrast, RT-2 samples with protruding $\mathrm{TiO}_{2}$ particles along the flake edge form relatively loosely stacked layers in films, due to $\mathrm{TiO}_{2}$ induced voids during the filtration process, leading to an increase in film thickness and decrease in packing density, which is one of the reasons for the reduction of both electrical conductivity and mechanical strength. 

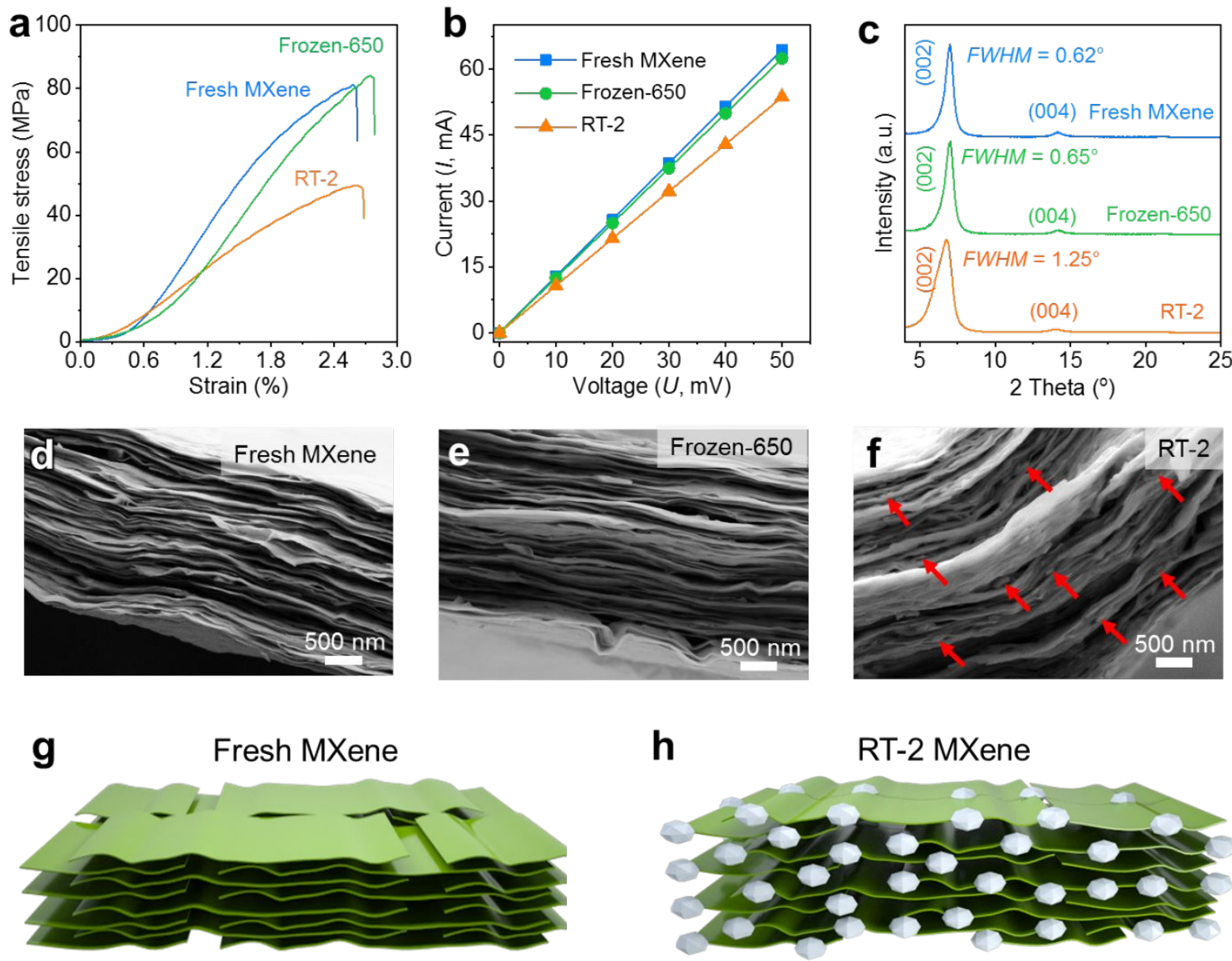

Figure 4. (a) The tensile-strain curves, (b) current-voltage (I-V) curves, (c) XRD spectra of MXene films produced from Fresh MXene, Frozen-650, and RT-2 dispersions. Scanning electron microscopy (SEM) photos of the cross-section of $\mathrm{Ti}_{3} \mathrm{C}_{2} \mathrm{~T}_{\mathrm{x}}$ films prepared using (d) Fresh MXene, (e) Frozen-650, and (f) RT-2 dispersions. Red arrows in (f) indicate the particles between MXene flakes. The structural models of $\mathrm{Ti}_{3} \mathrm{C}_{2} \mathrm{~T}_{\mathrm{x}}$ films prepared using (g) Fresh MXene and (h) RT-2 dispersions. The white $\mathrm{TiO}_{2}$ particles on $\mathrm{Ti}_{3} \mathrm{C}_{2} \mathrm{~T}_{\mathrm{x}}$ flakes working as spacers that increased the layer distance.

3.4. Electrochemical properties. To understand the electrochemical properties of Fresh MXene, RT-2 and Frozen-650 films, thin MXene films were prepared on glassy carbon electrodes 
and then assembled into a three-electrode test system in $1 \mathrm{M}$ of sulfuric acid electrolyte, using carbon rod and $\mathrm{Ag} / \mathrm{AgCl}$ electrode as counter and reference electrodes, respectively. All MXene electrodes show wide operation window of $-0.7 \mathrm{~V}$ to $0.2 \mathrm{~V}$, which is in agreement with previous works. ${ }^{10}$ Figure 5a-c show that all samples have the same curve shapes at low scan rate of $10 \mathrm{mV}$ $\mathrm{s}^{-1}$ with a peak at $\sim-0.37 \mathrm{~V}$, which can be attributed to the intercalation/de-intercalation of $\mathrm{H}^{+}$and surface redox reactions. ${ }^{46-47}$ For Fresh MXene and Frozen-650 films, the position of the peak current at $2 \mathrm{~V} \mathrm{~s}^{-1}$ shifted slightly to $-0.24 \mathrm{~V}$ and $-0.21 \mathrm{~V}$, respectively. However, at scan rate of 2 $\mathrm{V} \mathrm{s}^{-1}$ the RT-2 electrode shows a significant shift to $-0.05 \mathrm{~V}$. The RT-2 peak shift indicates the electrode has slower charge transfer rate than Fresh MXene and Frozen-650 electrodes. By integrating the area under the cyclic voltammetry (CV) curves, the specific capacitance changes of Fresh MXene, Frozen-650 and RT-2 electrodes were calculated as shown in Figure 5d. All electrodes showed the similar specific capacitance of $\sim 375 \mathrm{~F} \mathrm{~g} \mathrm{~g}^{-1}$ at low scan rate of $10 \mathrm{mV} \mathrm{s}^{-1}$. This result suggests that the early stage of oxidation has negligible impact on the electrode performance at low scan rate. The Frozen-650 shows the same changes in capacitance from $\sim 375 \mathrm{~F} \mathrm{~g}^{-1}$ at 10 $\mathrm{mV} \mathrm{s}^{-1}$ to $\sim 154 \mathrm{~F} \mathrm{~g}^{-1}$ at $10 \mathrm{~V} \mathrm{~s}^{-1}$, indicating the capacitance performance of Frozen-650 is as good as Fresh MXene. Otherwise, the specific capacitance of RT-2 sample dropped from $374 \mathrm{~F} \mathrm{~g}^{-1}$ at $10 \mathrm{mV} \mathrm{s}^{-1}$ to $\sim 81 \mathrm{~F} \mathrm{~g}^{-1}$ at $10 \mathrm{~V} \mathrm{~s}^{-1}$, which showed the rate performance is inhibited by MXene that has undergone Stage I oxidation. 

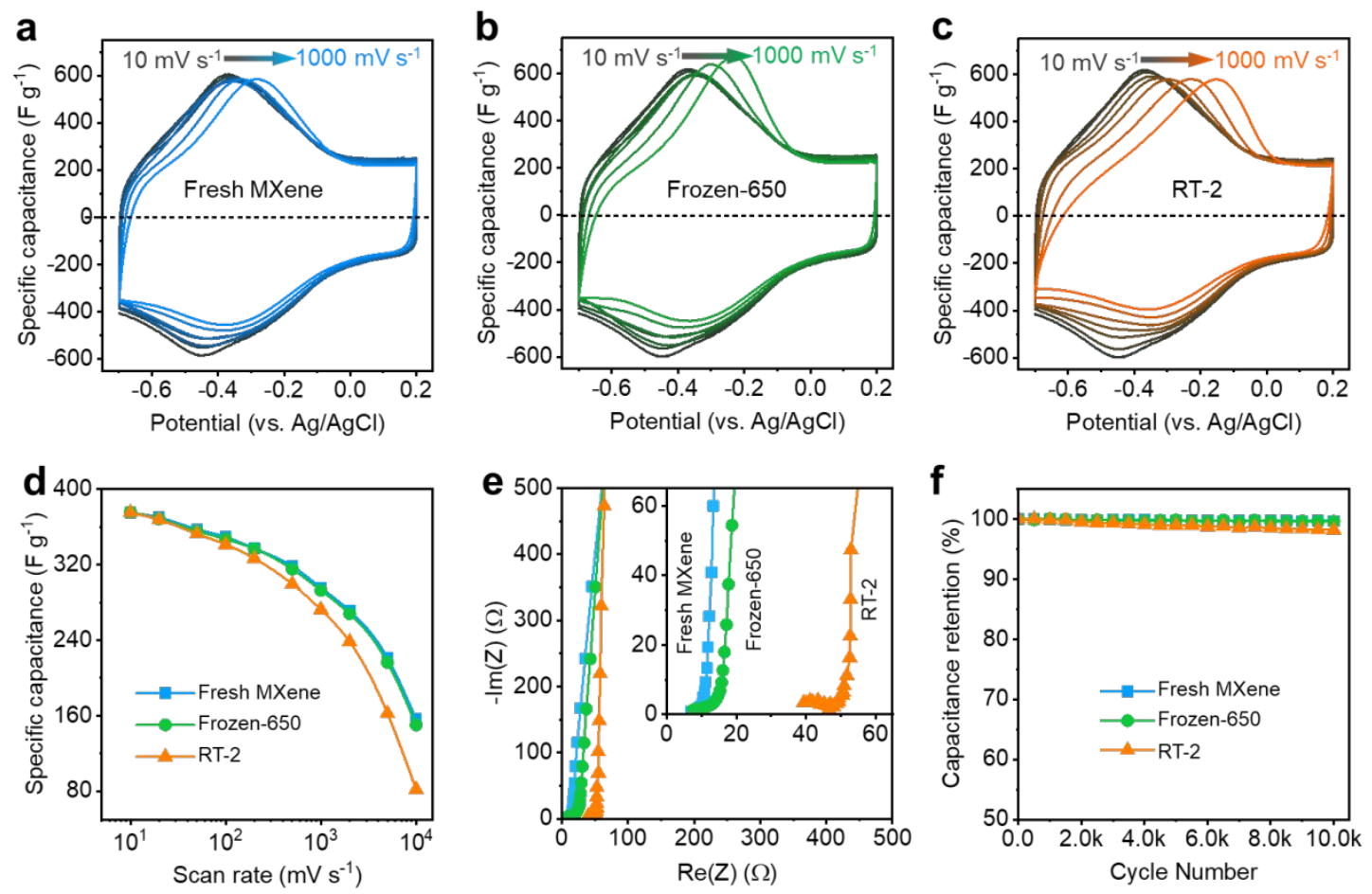

Figure 5. Cyclic voltammetry (CV) curves of (a) Fresh MXene, (b) Frozen-650 and (c) RT-2 electrodes in $1 \mathrm{M}$ sulfuric acid electrolyte at scan rates of $10 \mathrm{mV} \mathrm{s}^{-1}$ to $1000 \mathrm{mV} \mathrm{s}^{-1}$. (d) The changes of specific capacitance of electrodes as function of scan rate. (e) The Nyquist Plots of electrochemical impedance spectroscopy (EIS) data on above films. (f) Capacitance retention of Fresh MXene, Frozen-650 and RT-2 electrodes cycling at CV scan rate of $50 \mathrm{mV} \mathrm{s}^{-1}$.

To further understand the differences in the electrochemical properties of the fabricated films, we used electrochemical impedance spectroscopy (EIS) to study the above electrodes. The RT-2 film shows significant increase in the ohmic resistance $\left(R_{s}\right)$, which is in consistent with the reduced electrical conductivity due to oxidation (Figure 5e). The semi-circle in the high frequency region for RT-2, which corresponds to charge-transfer resistance $\left(R_{c t}\right)^{48-50}$, becomes larger compared with Fresh MXene and Frozen-650 films. In addition, all samples show quasi-vertical lines at low frequency ranging from around $100 \mathrm{mHz}$ to $1 \mathrm{~Hz}$, which are resulted from their highly capacitive 
behaviors. In the intermediate frequency region, the plots with their slope close to $45^{\circ}$, which appeared at $16.3 \mathrm{~Hz}$ and $14.7 \mathrm{~Hz}$ for the Fresh MXene and Frozen-650 films, respectively, can be attributed to the Warburg resistance originated from the semi-finite diffusion of ions in the electrodes. ${ }^{51-52}$ The observed Warburg frequency region decreased to $2.3 \mathrm{~Hz}$ for the RT-2 electrode, which suggests that Stage I oxidation hinders the ions diffusion in MXene electrode. The Fresh MXene and Frozen-650 electrodes exhibited good capacitance retention of $99.1 \%$ at a scan rate of $50 \mathrm{mV} \mathrm{s}^{-1}$ after 10,000 charging/discharging cycles, while the RT-2 electrode decreased to $98.3 \%$ (Figure $5 \mathrm{f}$ ). These results demonstrated the good long cycle stability of Frozen-650 electrodes.

Based from the evidences presented in this study, it can be inferred that there are three major reasons for the delay of early-stage oxidation of $\mathrm{Ti}_{3} \mathrm{C}_{2} \mathrm{~T}_{x}$ flakes. First, the lowered oxidation reaction rate constant resulted from a decreased storage temperature. Comparing the $4{ }^{\circ} \mathrm{C}$ and -20 ${ }^{\circ} \mathrm{C}$ sample, we found that $4{ }^{\circ} \mathrm{C}$ is suitable for storing MXene for less than 5 days, but for long-term storage, the liquid water has to be frozen in order to completely delay the oxidation of MXene flakes. Secondly, the ice formed around MXene flakes changed the media from liquid to solid in which ice crystals prevents the growth of $\mathrm{TiO}_{2}$ nanoparticles. ${ }^{13}$ The solid ice matrix surrounding the MXene flakes isolates said flakes from direct exposure to air. Additionally, the solid ice significantly decreases the diffusion rate of oxygen from the air to the MXene flakes. The third reason is that the amount of dissolved oxygen decreased when the aqueous dispersion was frozen into ice, reducing the contact of MXene flakes to oxygenated water molecules. Also, the solid ice can significantly decrease the diffusion rate of oxygen from air to MXene flakes. The combination of these three factors inhibits oxidation enabling long-term preservation of $\mathrm{Ti}_{3} \mathrm{C}_{2} \mathrm{~T}_{x}$ MXene dispersions through freezing. The present work revealed that the oxidation rate of MXene is 
influenced by temperature, which suggests it may follow the Arrhenius equation. However, more evidence is needed to prove that this is indeed the case and many other factors such as flake size, concentration, etc. need to be investigated to provide fundamental understanding to this Arrheniuslike behavior.

\section{CONCLUSION}

In this study, the temperature dependence of MXene oxidation in water was evaluated where a method to preserve $\mathrm{Ti}_{3} \mathrm{C}_{2} \mathrm{~T}_{x}$ MXene aqueous dispersions through freezing was developed. Assessment of morphology and properties of Fresh MXene, frozen MXene and MXene dispersions stored at room temperature, demonstrated that the $\mathrm{TiO}_{2}$ nanoparticles had already formed by the second day at room temperature while frozen dispersions showed minimal signs of oxidation. It is noteworthy that the MXene flakes stored by freezing also maintained same flake morphology and physical characteristics, such as electrical conductivity, mechanical properties and energy storage performance even after 650 days of storage. These results demonstrate that freezing MXene dispersions is a simple and effective method to enable long-term storage and transfer of MXene in aqueous dispersion.

\section{ASSOCIATED CONTENT}

Supporting Information. The Supporting Information is available free of charge on the ACS Publications website.

XRD pattern of $\mathrm{Ti}_{3} \mathrm{AlC}_{2}$ MAX phase and $\mathrm{Ti}_{3} \mathrm{C}_{2} \mathrm{~T}_{\mathrm{x}}$ MXene, photos of folded MXene film, and energy-dispersive X-ray spectroscopy (EDX) analysis of MXene films. (PDF)

\section{AUTHOR INFORMATION}

\section{Corresponding Author}


Prof. Joselito M. Razal (joselito.razal@deakin.edu.au)

\section{Author Contributions}

|| J. Zhang and N. Kong contributed equally to this manuscript. All authors have given approval to the final version of the manuscript.

\section{Notes}

There are no conflicts to declare.

\section{ACKNOWLEDGMENT}

The authors acknowledge financial support from the Australian Research Council (FT130100380 and IH140100018). The authors also acknowledge the Australian National Fabrication Facility (ANFF) Victorian nodes at La Trobe and Deakin Universities.

\section{REFERENCES}

(1) Naguib, M.; Mochalin, V. N.; Barsoum, M. W.; Gogotsi, Y. 25th Anniversary Article: MXenes: A New Family of Two-Dimensional Materials. Adv. Mater. 2014, 26, 992-1005.

(2) Deysher, G.; Shuck, C. E.; Hantanasirisakul, K.; Frey, N. C.; Foucher, A. C.; Maleski, K.; Sarycheva, A.; Shenoy, V. B.; Stach, E. A.; Anasori, B.; Gogotsi, Y. Synthesis of $\mathrm{Mo}_{4} \mathrm{VAlC}_{4} \mathrm{Max}$ Phase and Two-Dimensional Mo4vc4 MXene with Five Atomic Layers of Transition Metals. ACS Nano 2019, 14, 204-217.

(3) Peng, Q.; Guo, J.; Zhang, Q.; Xiang, J.; Liu, B.; Zhou, A.; Liu, R.; Tian, Y. Unique Lead Adsorption Behavior of Activated Hydroxyl Group in Two-Dimensional Titanium Carbide. J. Am. Chem. Soc. 2014, 136, 4113-4116.

(4) Hu, T.; Wang, J.; Zhang, H.; Li, Z.; Hu, M.; Wang, X. Vibrational Properties of $\mathrm{Ti}_{3} \mathrm{C}_{2}$ and $\mathrm{Ti}_{3} \mathrm{C}_{2} \mathrm{~T}_{2}(\mathrm{~T}=\mathrm{O}, \mathrm{F}, \mathrm{Oh})$ Monosheets by First-Principles Calculations: A Comparative Study. Phys. Chem. Chem. Phys. 2015, 17, 9997-10003.

(5) Karlsson, L. H.; Birch, J.; Halim, J.; Barsoum, M. W.; Persson, P. O. A. Atomically Resolved Structural and Chemical Investigation of Single MXene Sheets. Nano Lett. 2015, 15, 4955-4960.

(6) Hope, M. A.; Forse, A. C.; Griffith, K. J.; Lukatskaya, M. R.; Ghidiu, M.; Gogotsi, Y.; Grey, C. P. Nmr Reveals the Surface Functionalisation of $\mathrm{Ti}_{3} \mathrm{C}_{2}$ MXene. Phys. Chem. Chem. Phys. 2016, 18, 5099-5102.

(7) Wang, H.-W.; Naguib, M.; Page, K.; Wesolowski, D. J.; Gogotsi, Y. Resolving the Structure of $\mathrm{Ti}_{3} \mathrm{C}_{2} \mathrm{~T}_{\mathrm{x}}$ MXenes through Multilevel Structural Modeling of the Atomic Pair Distribution Function. Chem. Mater. 2016, 28, 349-359. 
(8) Naguib, M.; Mashtalir, O.; Carle, J.; Presser, V.; Lu, J.; Hultman, L.; Gogotsi, Y.; Barsoum, M. W. Two-Dimensional Transition Metal Carbides. ACS Nano 2012, 6, 1322-1331.

(9) Ghidiu, M.; Lukatskaya, M. R.; Zhao, M.-Q.; Gogotsi, Y.; Barsoum, M. W. Conductive TwoDimensional Titanium Carbide 'Clay' with High Volumetric Capacitance. Nature 2014, 516, 78U171.

(10) Lukatskaya, M. R.; Kota, S.; Lin, Z.; Zhao, M.-Q.; Shpigel, N.; Levi, M. D.; Halim, J.; Taberna, P.-L.; Barsoum, M. W.; Simon, P.; Gogotsi, Y. Ultra-High-Rate Pseudocapacitive Energy Storage in Two-Dimensional Transition Metal Carbides. Nat. Energy 2017, 6, 17105.

(11) Zhang, J.; Kong, N.; Uzun, S.; Levitt, A.; Seyedin, S.; Lynch, P. A.; Qin, S.; Han, M.; Yang, W.; Liu, J.; Wang, X.; Gogotsi, Y.; Razal, J. M. Scalable Manufacturing of Free-Standing, Strong $\mathrm{Ti}_{3} \mathrm{C}_{2} \mathrm{~T}_{\mathrm{x}}$ MXene Films with Outstanding Conductivity. Adv. Mater. 2020, 32, 2001093.

(12) Zhang, C. J.; Pinilla, S.; McEvoy, N.; Cullen, C. P.; Anasori, B.; Long, E.; Park, S.-H.; SeralAscaso, A.; Shmeliov, A.; Krishnan, D.; Morant, C.; Liu, X.; Duesberg, G. S.; Gogotsi, Y.; Nicolosi, V. Oxidation Stability of Colloidal Two-Dimensional Titanium Carbides (MXenes). Chem. Mater. 2017, 29, 4848-4856.

(13) Habib, T.; Zhao, X.; Shah, S. A.; Chen, Y.; Sun, W.; An, H.; Lutkenhaus, J. L.; Radovic, M.; Green, M. J. Oxidation Stability of $\mathrm{Ti}_{3} \mathrm{C}_{2} \mathrm{~T}_{\mathrm{x}}$ MXene Nanosheets in Solvents and Composite Films. npj 2D Mater. Appl. 2019, 3, 8.

(14) Lipatov, A.; Alhabeb, M.; Lukatskaya, M. R.; Boson, A.; Gogotsi, Y.; Sinitskii, A. Effect of Synthesis on Quality, Electronic Properties and Environmental Stability of Individual Monolayer $\mathrm{Ti}_{3} \mathrm{C}_{2}$ MXene Flakes. Adv. Electron Mater. 2016, 2, 1600255.

(15) Cao, M.; Wang, F.; Wang, L.; Wu, W.; Lv, W.; Zhu, J. Room Temperature Oxidation of $\mathrm{Ti}_{3} \mathrm{C}_{2}$ MXene for Supercapacitor Electrodes. J. Electrochem. Soc. 2017, 164, A3933-A3942.

(16) Peng, C.; Yang, X.; Li, Y.; Yu, H.; Wang, H.; Peng, F. Hybrids of Two-Dimensional $\mathrm{Ti}_{3} \mathrm{C}_{2}$ and $\mathrm{TiO}_{2}$ Exposing $\{001\}$ Facets toward Enhanced Photocatalytic Activity. ACS Appl. Mater. Interfaces. 2016, 8, 6051-6060.

(17) Naguib, M.; Mashtalir, O.; Lukatskaya, M. R.; Dyatkin, B.; Zhang, C.; Presser, V.; Gogotsi, Y.; Barsoum, M. W. One-Step Synthesis of Nanocrystalline Transition Metal Oxides on Thin Sheets of Disordered Graphitic Carbon by Oxidation of MXenes. Chem. Commun. 2014, 50, 74207423.

(18) Seyedin, S.; Zhang, J.; Usman, K. A. S.; Qin, S.; Glushenkov, A. M.; Yanza, E. R. S.; Jones, R. T.; Razal, J. M. Facile Solution Processing of Stable MXene Dispersions Towards Conductive Composite Fibers. Glob. Chall. 2019, 3, 1900037.

(19) Wang, C.-H.; Kurra, N.; Alhabeb, M.; Chang, J.-K.; Alshareef, H. N.; Gogotsi, Y. Titanium Carbide (MXene) as a Current Collector for Lithium-Ion Batteries. ACS Omega 2018, 3, 1248912494.

(20) Zhang, J.; Uzun, S.; Seyedin, S.; Lynch, P. A.; Akuzum, B.; Wang, Z.; Qin, S.; Alhabeb, M.; Shuck, C. E.; Lei, W.; Kumbur, E. C.; Yang, W.; Wang, X.; Dion, G.; Razal, J. M.; Gogotsi, Y. Additive-Free MXene Liquid Crystals and Fibers. ACS Cent. Sci. 2020, 6, 254-265.

(21) Zhang, Y.-Z.; Lee, K. H.; Anjum, D. H.; Sougrat, R.; Jiang, Q.; Kim, H.; Alshareef, H. N. MXenes Stretch Hydrogel Sensor Performance to New Limits. Sci. Adv. 2018, 4, eaat0098.

(22) Han, M.; Yin, X.; Hantanasirisakul, K.; Li, X.; Iqbal, A.; Hatter, C. B.; Anasori, B.; Koo, C. M.; Torita, T.; Soda, Y.; Zhang, L.; Cheng, L.; Gogotsi, Y. Anisotropic MXene Aerogels with a Mechanically Tunable Ratio of Electromagnetic Wave Reflection to Absorption. Adv. Opt. Mater. 2019, 7, 1900267. 
(23) Xia, Y.; Mathis, T. S.; Zhao, M.-Q.; Anasori, B.; Dang, A.; Zhou, Z.; Cho, H.; Gogotsi, Y.; Yang, S. Thickness-Independent Capacitance of Vertically Aligned Liquid-Crystalline MXenes. Nature 2018, 557, 409-412.

(24) Maleski, K.; Mochalin, V. N.; Gogotsi, Y. Dispersions of Two-Dimensional Titanium Carbide MXene in Organic Solvents. Chem. Mater. 2017, 29, 1632-1640.

(25) Zhao, X.; Vashisth, A.; Prehn, E.; Sun, W.; Shah, S. A.; Habib, T.; Chen, Y.; Tan, Z.; Lutkenhaus, J. L.; Radovic, M.; Green, M. J. Antioxidants Unlock Shelf-Stable $\mathrm{Ti}_{3} \mathrm{C}_{2} \mathrm{~T}_{\mathrm{x}}$ (MXene) Nanosheet Dispersions. Matter 2019, 1, 513-526.

(26) Levitt, A. S.; Alhabeb, M.; Hatter, C.; Sarycheva, A.; Dion, G.; Gogotsi, Y. Electrospun MXene/Carbon Nanofibers as Supercapacitor Electrodes. J. Mater. Chem. A 2018, 7, 269-277.

(27) Seyedin, S.; Yanza, E.; Razal, J. M. Knittable Energy Storing Fiber with High Volumetric Performance Made from Predominantly MXene Nanosheets. J. Mater. Chem. A 2017, 5, 2407624082.

(28) Zhang, J.; Seyedin, S.; Qin, S.; Wang, Z.; Moradi, S.; Yang, F.; Lynch, P. A.; Yang, W.; Liu, J.; Wang, X.; Razal, J. M. Highly Conductive $\mathrm{Ti}_{3} \mathrm{C}_{2} \mathrm{~T}_{\mathrm{x}}$ MXene Hybrid Fibers for Flexible and Elastic Fiber-Shaped Supercapacitors. Small 2019, 15, 1804732.

(29) Mashtalir, O.; Naguib, M.; Mochalin, V. N.; Dall'Agnese, Y.; Heon, M.; Barsoum, M. W.; Gogotsi, Y. Intercalation and Delamination of Layered Carbides and Carbonitrides. Nat. Commun. 2013, 4, 1716.

(30) Lee, Y.; Kim, S. J.; Kim, Y.-J.; Lim, Y.; Chae, Y.; Lee, B.-J.; Kim, Y.-T.; Han, H.; Gogotsi, Y.; Ahn, C. W. Oxidation-Resistant Titanium Carbide MXene Films. J. Mater. Chem. A 2020, 8, 573-581.

(31) Rubinsky, B. Principles of Low Temperature Cell Preservation. Heart Fail. Rev. 2003, 8, 277 284.

(32) Fennema, O.; Powrie, W. D. Fundamentals of Low-Temperature Food Preservation. In Advances in Food Research; Chichester, C. O.; Mrak, E. M.; Stewart, G. F., Eds.; Academic Press: 1964; pp 219-347.

(33) Roth, J.; Bendayan, M.; Carlemalm, E.; Villiger, W.; Garavito, M. Enhancement of Structural Preservation and Immunocytochemical Staining in Low Temperature Embedded Pancreatic Tissue. J. Histochem. Cytochem. 1981, 29, 663-671.

(34) White, I. Lipids and Calcium Uptake of Sperm in Relation to Cold Shock and Preservation: A Review. Reproduction Fertil. Dev. 1993, 5, 639-658.

(35) Chae, Y.; Kim, S. J.; Cho, S. Y.; Choi, J.; Maleski, K.; Lee, B. J.; Jung, H. T.; Gogotsi, Y.; Lee, Y.; Ahn, C. W. An Investigation into the Factors Governing the Oxidation of TwoDimensional $\mathrm{Ti}_{3} \mathrm{C}_{2}$ MXene. Nanoscale 2019, 11, 8387-8393.

(36) Shahzad, F.; Alhabeb, M.; Hatter, C. B.; Anasori, B.; Man Hong, S.; Koo, C. M.; Gogotsi, Y. Electromagnetic Interference Shielding with 2D Transition Metal Carbides (MXenes). Science 2016, 353, 1137-1140.

(37) Peng, Y.-Y.; Akuzum, B.; Kurra, N.; Zhao, M.-Q.; Alhabeb, M.; Anasori, B.; Kumbur, E. C.; Alshareef, H. N.; Ger, M.-D.; Gogotsi, Y. All-MXene (2D Titanium Carbide) Solid-State Microsupercapacitors for on-Chip Energy Storage. Energy Environ. Sci. 2016, 9, 2847-2854.

(38) Malaki, M.; Maleki, A.; Varma, R. S. MXenes and Ultrasonication. J. Mater. Chem. A 2019, 7, 10843-10857.

(39) Ghassemi, H.; Harlow, W.; Mashtalir, O.; Beidaghi, M.; Lukatskaya, M. R.; Gogotsi, Y.; Taheri, M. L. In Situ Environmental Transmission Electron Microscopy Study of Oxidation of 
Two-Dimensional $\mathrm{Ti}_{3} \mathrm{C}_{2}$ and Formation of Carbon-Supported $\mathrm{TiO}_{2}$. J. Mater. Chem. A 2014, 2, 14339-14343.

(40) Shuck, C. E.; Han, M.; Maleski, K.; Hantanasirisakul, K.; Kim, S. J.; Choi, J.; Reil, W. E. B.; Gogotsi, Y. Effect of $\mathrm{Ti}_{3} \mathrm{AlC}_{2}$ MAX Phase on Structure and Properties of Resultant $\mathrm{Ti}_{3} \mathrm{C}_{2} \mathrm{~T}_{\mathrm{x}}$ MXene. ACS Appl. Nano Mater. 2019, 2, 3368-3376.

(41) Lipatov, A.; Lu, H.; Alhabeb, M.; Anasori, B.; Gruverman, A.; Gogotsi, Y.; Sinitskii, A. Elastic Properties of 2D $\mathrm{Ti}_{3} \mathrm{C}_{2} \mathrm{~T}_{\mathrm{x}}$ MXene Monolayers and Bilayers. Sci. Adv. 2018, 4, eaat0491.

(42) Yan, J.; Ren Chang, E.; Maleski, K.; Hatter Christine, B.; Anasori, B.; Urbankowski, P.; Sarycheva, A.; Gogotsi, Y. Flexible MXene/Graphene Films for Ultrafast Supercapacitors with Outstanding Volumetric Capacitance. Adv. Funct. Mater. 2017, 27, 1701264.

(43) Halim, J.; Cook, K. M.; Naguib, M.; Eklund, P.; Gogotsi, Y.; Rosen, J.; Barsoum, M. W. XRay Photoelectron Spectroscopy of Select Multi-Layered Transition Metal Carbides (MXenes). Appl. Surf. Sci. 2016, 362, 406-417.

(44) Rasheed, P. A.; Pandey, R. P.; Rasool, K.; Mahmoud, K. A. Ultra-Sensitive Electrocatalytic Detection of Bromate in Drinking Water Based on Nafion/ $\mathrm{Ti}_{3} \mathrm{C}_{2} \mathrm{~T}_{\mathrm{x}}$ (MXene) Modified Glassy Carbon Electrode. Sens. Actuators B Chem. 2018, 265, 652-659.

(45) Low, J.; Zhang, L.; Tong, T.; Shen, B.; Yu, J. TiO 2 MXene $\mathrm{Ti}_{3} \mathrm{C}_{2}$ Composite with Excellent Photocatalytic $\mathrm{Co}_{2}$ Reduction Activity. J. Catal. 2018, 361, 255-266.

(46) Lukatskaya, M. R.; Mashtalir, O.; Ren, C. E.; Dall'Agnese, Y.; Rozier, P.; Taberna, P. L.; Naguib, M.; Simon, P.; Barsoum, M. W.; Gogotsi, Y. Cation Intercalation and High Volumetric Capacitance of Two-Dimensional Titanium Carbide. Science 2013, 341, 1502-1505.

(47) Lukatskaya, M. R.; Bak, S.-M.; Yu, X.; Yang, X.-Q.; Barsoum, M. W.; Gogotsi, Y. Probing the Mechanism of High Capacitance in 2D Titanium Carbide Using in Situ X-Ray Absorption Spectroscopy. Adv. Energy Mater. 2015, 5, 1500589.

(48) Lefebvre, M.; Qi, Z. G.; Rana, D.; Pickup, P. G. Chemical Synthesis, Characterization, and Electrochemical Studies of Poly(3,4-Ethylenedioxythiophene)/Poly(Styrene-4-Sulfonate) Composites. Chem. Mater. 1999, 11, 262-268.

(49) Taberna, P. L.; Portet, C.; Simon, P. Electrode Surface Treatment and Electrochemical Impedance Spectroscopy Study on Carbon/Carbon Supercapacitors. Appl. Phys. A Mater. 2006, $82,639-646$.

(50) Benavente, J. Electrochemical Impedance Spectroscopy as a Tool for Electrical and Structural Characterizations of Membranes in Contact with Electrolyte Solutions. In Recent Advances in Multidisciplinary Applied Physics; Méndez-Vilas, A., Ed.; Elsevier Science Ltd: Oxford, 2005; pp 463-471.

(51) Xia, Q. X.; Fu, J.; Yun, J. M.; Mane, R. S.; Kim, K. H. High Volumetric Energy Density Annealed-MXene-Nickel Oxide/MXene Asymmetric Supercapacitor. $R S C A d v$. 2017, 7, 1100011011.

(52) Lin, Z.; Barbara, D.; Taberna, P.-L.; Van Aken, K. L.; Anasori, B.; Gogotsi, Y.; Simon, P. Capacitance of $\mathrm{Ti}_{3} \mathrm{C}_{2} \mathrm{~T}_{\mathrm{x}}$ MXene in Ionic Liquid Electrolyte. J. Power Sources 2016, 326, 575-579. 
TOC:

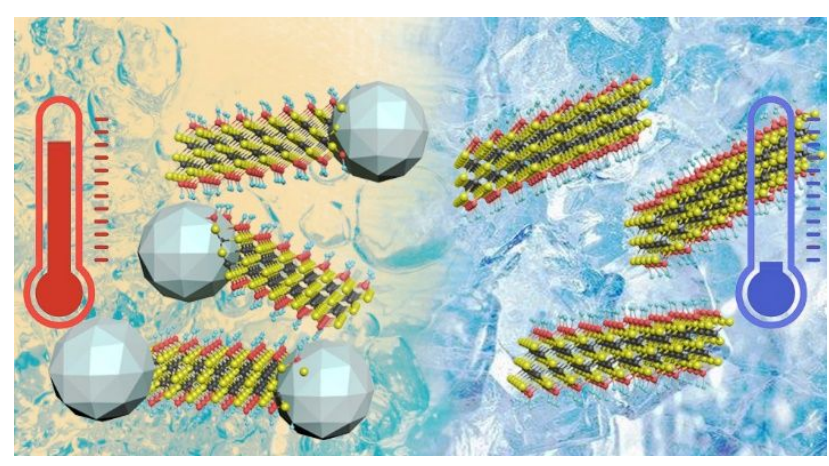

